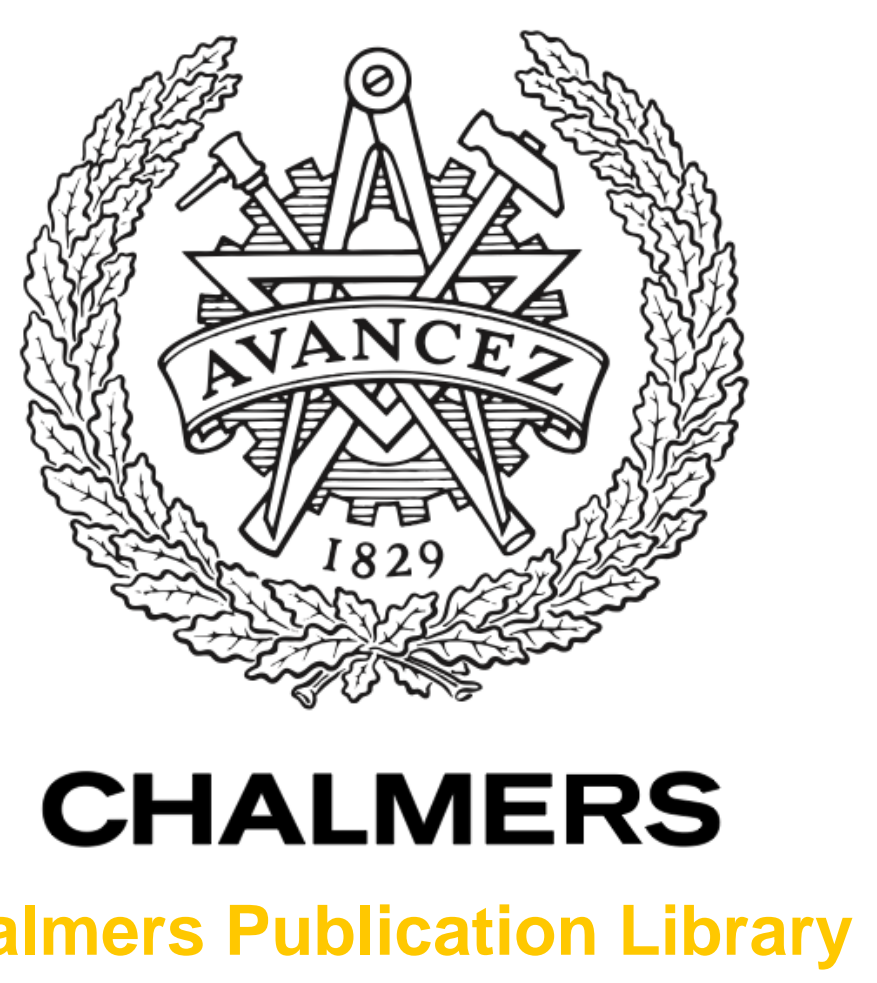

Chalmers Publication Library

\title{
Predicting the Solubility of Sulfur: A COSMO-RS-Based Approach to Investigate Electrolytes for Li-S Batteries
}

This document has been downloaded from Chalmers Publication Library (CPL). It is the author's version of a work that was accepted for publication in:

Chemistry-a European Journal (ISSN: 0947-6539)

Citation for the published paper:

Jeschke, S. ; Johansson, P. (2017) "Predicting the Solubility of Sulfur: A COSMO-RS-Based Approach to Investigate Electrolytes for Li-S Batteries". Chemistry-a European Journal, vol. 23(38), pp. 9130-9136.

http://dx.doi.org/10.1002/chem.201701011

Downloaded from: http://publications.lib.chalmers.se/publication/251082

Notice: Changes introduced as a result of publishing processes such as copy-editing and formatting may not be reflected in this document. For a definitive version of this work, please refer to the published source. Please note that access to the published version might require a subscription.

Chalmers Publication Library (CPL) offers the possibility of retrieving research publications produced at Chalmers University of Technology. It covers all types of publications: articles, dissertations, licentiate theses, masters theses, conference papers, reports etc. Since 2006 it is the official tool for Chalmers official publication statistics. To ensure that Chalmers research results are disseminated as widely as possible, an Open Access Policy has been adopted.

The CPL service is administrated and maintained by Chalmers Library. 


\title{
Predicting solubility of sulfur: A COSMO-RS based approach to investigate electrolytes for Li-S batteries
}

\author{
Steffen Jeschke ${ }^{*[a]}$ and Patrik Johansson ${ }^{[a]}$
}

\begin{abstract}
Lithium-sulfur (Li-S) batteries are in theory, from their basic reactions, very promising from a specific energy density point-of-view, but have bad power rate capabilities. The dissolution of sulfur from the $\mathrm{C} / \mathrm{S}$ cathodes into the electrolyte is a rate determining and crucial step for the functionality. So far, time-consuming experimental methods, such as HPLC/UV, have been used to quantify the corresponding solubilities. Here, we use a computational fluid phase thermodynamics approach, the conductor-like screening model for real solvents (COSMO-RS), to compute the solubility of sulfur in different binary and ternary electrolytes. By using both explicit and implicit solvation approaches for LiTFSI containing electrolytes a deviation $<0.4 \log$ units was achieved $v s$. experimental data - in the range of experimental error and hence proves COSMO-RS to be a tool for exploring novel Li-S battery electrolytes.
\end{abstract}

\section{Introduction}

The lithium-sulfur battery is considered as one possible next generation battery for energy storage in electric vehicles. Using sulfur as the active electrode material is advantageous as it is abundant, environmentally friendly, and cheaper than any current cathode materials. Also, it has a high theoretical capacity of $1672 \mathrm{mAh} \mathrm{g}^{-1}$ and an energy density of $\sim 2500 \mathrm{Wh} \mathrm{kg}^{-1}$, assuming a complete reduction to $\mathrm{Li}_{2} \mathrm{~S}{ }^{[1,2]}$ Due to its insulating nature the cathode is often a combination with porous carbon materials $(\mathrm{C} / \mathrm{S})$. During discharge, sulfur is reduced to various intermediate polysulfides such as $\mathrm{S}_{8}{ }^{2-}, \mathrm{S}_{6}{ }^{2-}, \mathrm{S}_{4}{ }^{2-}$, and $\mathrm{S}_{2}{ }^{2-}$ before $\mathrm{S}^{2-}$ is reached, and the process is ideally reversible and the sulfides are oxidized back to sulfur during charging. However, Li-S batteries suffer from poor cyclability mainly due to the solubility of elemental sulfur and polysulfides into the electrolyte causing loss of active material. This results in capacity fading, increase in internal resistance, poor coulombic efficiencies, and self-discharge. Assuming that elemental sulfur dissolves prior to reduction and hence energy production, suppressing and controlling the solubility of sulfur is a given approach to improve cycling stability. ${ }^{[2-4]}$

In order to quantify the solubility of sulfur in various conventional Li-S battery liquid organic electrolytes, often binary or ternary systems, ${ }^{[4]}$ high performance liquid chromatography coupled with

[a] Dr. S. Jeschke, Prof. P. Johansson

Department of Physics

Chalmers University of Technology

41296 Gothenburg (Sweden)

E-mail: steffen.jeschke@chalmers.se

Supporting information for this article (further dataset for calculation of $\Delta$ fus $G$, COSMO-RS $\sigma$-profiles of investigated solvents, calculated excess properties) is given via a link at the end of the document: a UV detector (HPLC/UV) ${ }^{[5,6]}$ has been used. The application of supportive computational methods and predictive models are useful in reducing the experimental work needed for further and optimized solvent selection, but has not yet been reported for screening of electrolytes for Li-S batteries.

In this context, we here apply the conductor-like screening model for real solvents (COSMO-RS), developed by Klamt ${ }^{[7]}$ to compute physical properties of mixed liquid phases, such as vaporpressure ${ }^{[8,9]}$ partition and activity coefficients, ${ }^{[10]}$ phase equilibria prediction of pKa-values ${ }^{[11,12]}$ and redox-potentials, ${ }^{[13,14]}$ for Li-S battery solvents and electrolytes. COSMO-RS combines a quantum chemically (DFT) based continuum solvation approach for a single molecule with statistical thermodynamics and has already proven to be an effective and reliable screening tool when it comes to pharmaceutical drugs, ${ }^{[15,16]}$ industrial separation thermodynamics ${ }^{[17]}$ and ionic liquids. ${ }^{[18-20]}$

Herein, we present the first study on applying COSMO-RS as a computational screening tool during the design of novel electrolytes for Li-S batteries. A three-step approach is chosen: (I) referencing the COSMO-RS performance to sulfur solubility in organic solvents, (II) comparing the computed sulfur solubilities for binary and ternary electrolytes with experimental data, and (III) analysis of the thermodynamic properties related to the

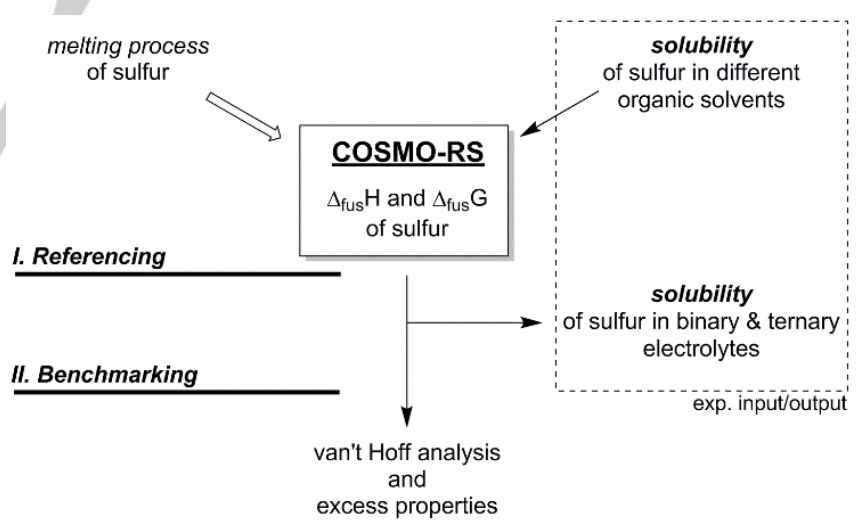

II. Thermodynamic analysis

dissolution process (Figure 1).

Figure 1. Conceptual approach of COSMO-RS as a computational support for Li-S battery electrolyte screening. For sulfur, only the cyclo-S $\mathrm{S}_{8}$ allotrope was considered.

As COSMO-RS was originally developed for neutral compounds or organic ions, such as the TFSI anion, alkali ions such as $\mathrm{Li}^{+}$, with a high charge density, might result in significant errors as the polarization charge density cannot be compensated by the polarization charge of the solvent, ultimately causing a misfit 
charge in the COSMO-RS thermodynamics. ${ }^{[1]}$ Furthermore, the long-range interactions between ions in dilute electrolytes, as considered in Debye-Hückel theory, are in reality contributing, but not accounted for in COSMO-RS. ${ }^{[22,23]}$ To resolve these shortcomings we use organic solvents in step (I) and two different methods to describe the binary and ternary electrolytes in step (II): (A): an implicit solvation of $\mathrm{Li}^{+}$, and (B): an explicit solvation of $\mathrm{Li}^{+}$, where it is completely replaced by corresponding solvent and anion based clusters. Hereby we can also evaluate the effect of the description of the electrolyte at the molecular level, both in terms of accuracy and computational effort.

\section{Results and Discussion}

\section{Referencing}

As COSMO-RS has been developed to compute miscibility and physical properties of liquid phases solely, the calculation of the solubility of sulfur was divided in two steps: (a) the virtual transfer of the solid sulfur in a supercooled liquid and (b) mixing of supercooled liquid sulfur with the solvent. For step (a) the molar free energy of fusion ( $\Delta_{\text {fus }} G$ ) is required, while for step (b) the chemical potentials of pure sulfur $\left(\mu_{s}\right)$ and sulfur solvated in a solvent $\left(\mu_{S}\right.$ soly $)$ are needed and are computed with high accuracy in COSMO-RS. As a result, the mole fraction solubility of sulfur $\left(x_{S}\right)$ can be obtained via

$\ln \left(x_{S}\right)=\frac{\mu_{S^{-}} \mu_{S}^{\text {sol }}-\Delta_{f u s} G}{R T}$

where $T$ is the temperature and $R$ is the gas constant. According to eq. $1, \Delta$ fus $G$ can either be provided experimentally to predict $X_{s}$, or vice versa. As an intrinsic property, $\Delta$ fus $G$ should be identical as long as the virtual as well as the real description of the investigated solute are identical. Here, elemental sulfur has been simplified to $c y c l o-S_{8}$, being the most well-known allotrope of sulfur ${ }^{[2,25]}$ and hence the 'virtual' or 'computed' sulfur is 'pure' whereas 'real' sulfur contains 'impurities' in terms of various allotropes. As a result, the $\Delta_{\text {fus }} G$ value obtained from the experimental thermal analysis, $3.6 \mathrm{~kJ} \mathrm{~mol}^{-1}$, and those computed by COSMO-RS from experimental solubilities are expected to differ.

Table 1. Experimental sulfur solubility in different organic solvents ${ }^{[5]}$ and the corresponding calculated molar free energy of fusion $\left(\Delta_{\text {fus }} G\right)$.

\begin{tabular}{lccc}
\hline solvent & density $/ \mathrm{g} \mathrm{cm}^{-3}$ & solubility $/ \mathrm{mM}$ & $\Delta_{\text {fus }} \mathrm{G} / \mathrm{kJ} \mathrm{mol}^{-1}$ \\
\hline ACN & 0.786 & 0.61 & 19.7 \\
Ace & 0.784 & 2.11 & 15.6 \\
DMSO & 1.100 & 3.94 & 9.0 \\
DMF & 0.944 & 5.94 & 13.0 \\
Hex & 0.655 & 7.07 & 10.8 \\
EtOH & 0.789 & 1.70 & 11.0
\end{tabular}

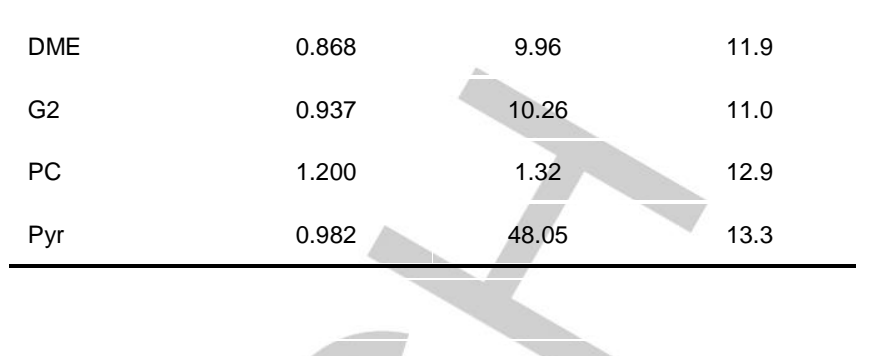

Based on the solubility of sulfur in 10 different organic solvents of different polarity by Zheng et al., ${ }^{[5]} \Delta_{\text {fus }} G$ was calculated to $9.0-$ $15.5 \mathrm{~kJ} \mathrm{~mol}^{-1}$; average: $12.1 \mathrm{~kJ} \mathrm{~mol}^{-1}$ for nine of the solvents, but $19.1 \mathrm{~kJ} \mathrm{~mol}^{-1}$ for acetonitrile (Table 1) - a solvent henceforth excluded. Additionally, a second dataset with experimental solubilities of sulfur in 15 different organic solvents is provided (Table S1). ${ }^{[26]}$ From these an average $\Delta$ fus $G$ of $11.3 \mathrm{~kJ} \mathrm{~mol}^{-1}$ was obtained from 13 of the solvents, while two solvents seem to have an overestimated solubility. However, this second dataset dates back to the 1950's with unknown experimental procedures, why henceforth a $\Delta_{\text {fus }} G$ of $12.1 \mathrm{~kJ} \mathrm{~mol}^{-1}\left(2.9 \mathrm{kcal} \mathrm{mol}^{-1}\right)$ will be used. The simplification to cyclo-S $\mathrm{S}_{8}$ in the model, as compared to the experimental real allotrope-mixed sulfur, results in a higher $\Delta$ fus $G$ computed, from either dataset of experimental solubilities.

\section{Benchmarking \\ Binary electrolytes}

In order to verify the reliability of the COMSO-RS approach, the solubility of cyclo- $\mathrm{S}_{8}$ in binary electrolytes with $0.1 \mathrm{M}$ and $1.0 \mathrm{M}$ LiTFSI were computed and compared to the literature..$^{[5]}$ For the implicit solvation approach (A) three components; solvent, $\mathrm{Li}^{+}$and TFSI, were considered and their mole fractions were calculated directly using the density of the corresponding organic solvent (Table 1).

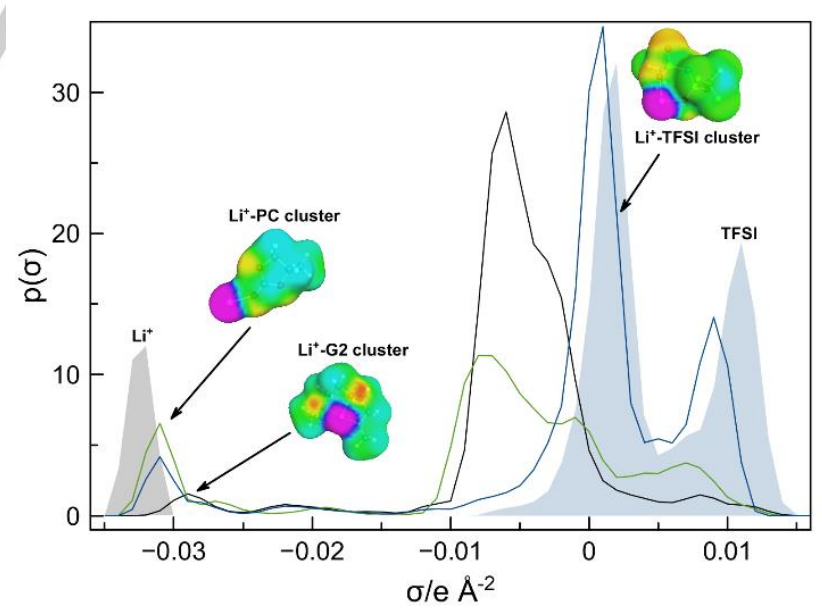

Figure 2. $\sigma$-profiles of different $\mathrm{Li}^{+}$-solvent clusters. In the COSMO-spheres, a red area denotes a negatively charged part of the molecular surface and hence 
a positive charge density $\sigma$. A purple area denotes strongly positively charged surface regions and a green area denotes non-polar surface regions.

The explicit solvation approach (B) reduces the peak of the "free" cation and spreads out the $\sigma$-profile closer to the $\sigma$-range of -0.02 e $\AA^{-2}$ (Figure 2). To maintain constant solvent:LiTFSI ratios in both approaches $(A)$ and $(B)$, the content of the different clusters in (B) was predicted from an intermolecular contact statistics calculation for $(\mathrm{A})$. The $\mathrm{Li}^{+}$is exclusively contacted by a solvent molecule in the $0.1 \mathrm{M} \mathrm{LiTFSI}$ electrolytes, which corresponds to a fully dissociated LiTFSI salt (Table 2). The contact statistics calculation is, however, based on the pairwise pairing of molecular surface segments and therefore describes formation of $\left[\mathrm{Li}\left(\right.\right.$ solvent)] ${ }^{+}$-clusters rather than the full cation solvation shell. Accordingly, the formation of a [Li(TFSI)]-cluster becomes more likely for the $1.0 \mathrm{M}$ LiTFSI electrolytes. The formation of [Li(solvent)] ${ }^{+}$-clusters is more likely for DMF, DMSO, or Pyr as solvents than for DME, G2, or PC, due to the charge distributions; in DMF its mesomeric $>\mathrm{N}^{+}=\mathrm{C}-\mathrm{O}^{-}$form, in DMSO its $>\mathrm{S}^{+}-\mathrm{O}^{-}$form, and in Pyr the localized lone pair on the nitrogen atom, each representing predominant sites of interaction for the lithium cation. Any potential bidentate coordination of $\mathrm{Li}^{+}$by $\mathrm{DME}$ or $\mathrm{G} 2$ is not accounted for in the contact statistics calculations. Subsequently, the mole fractions of all components in a binary electrolyte were recalculated and the cyclo- $S_{8} /$ binary electrolyte solid-liquid equilibrium was computed to obtain the maximum cyclo- $S_{8}$ mole fraction solubility $x_{s}$.

Table 2. Contact probability for $\mathrm{Li}^{+}$in binary electrolytes.

\begin{tabular}{|c|c|c|c|c|c|c|}
\hline & DMF & DMSO & DME & G2 & $\mathrm{PC}$ & Pyr \\
\hline \multicolumn{7}{|c|}{0.1 M LiTFSI electrolyte } \\
\hline solvent & 1.00 & 1.00 & 0.99 & 0.99 & 0.99 & 0.99 \\
\hline TFSI & 0.00 & 0.00 & 0.01 & 0.01 & 0.01 & 0.01 \\
\hline \multicolumn{7}{|c|}{1.0 M LiTFSI electrolyte } \\
\hline solvent & 0.89 & 0.93 & 0.80 & 0.81 & 0.83 & 0.87 \\
\hline TFSI & 0.11 & 0.07 & 0.20 & 0.19 & 0.17 & 0.13 \\
\hline
\end{tabular}

Table 3 shows the significant improvement in calculated solubilities for the $0.1 \mathrm{M}$ LiTFSI binary electrolyte by using the explicit solvation approach, reducing the RMSD from 0.8 to 0.4 log units. For the 1.0 M LiTFSI binary electrolyte both approaches are similarly accurate, but with a slightly better performance of the implicit solvation approach. For most electrolytes the cyclo- $\mathrm{S}_{8}$ solubility tends to be overestimated, the exception is the DMSO based electrolytes. For neat DMSO, a cyclo- $\mathrm{S}_{8} \Delta$ fus $\mathrm{G}$-value of $9.0 \mathrm{~kJ} \mathrm{~mol}^{-1}$ has been determined above, which is ca. $3 \mathrm{~kJ} \mathrm{~mol}^{-1}$ lower than the used mean, hence the solubility might be underestimated due to the larger energy required for the virtual melting process.
Table 3. Comparison of experimental[5] (exp.) sulfur and calculated cyclo-S 8 solubilities in various solvents according to the different solvation approaches (implicit: A; explicit: B).

\begin{tabular}{|c|c|c|c|c|c|c|c|c|}
\hline \multirow[b]{2}{*}{ solvent } & \multirow[b]{2}{*}{$\begin{array}{l}\text { solu./ } \\
\mathrm{mM}\end{array}$} & \multicolumn{3}{|c|}{$\begin{array}{c}\log _{10}\left(x_{S}\right) \text { for } 0.1 \mathrm{M} \\
\text { LiTFSI }\end{array}$} & \multirow[b]{2}{*}{$\begin{array}{l}\text { solu./ } \\
\mathrm{mM}\end{array}$} & \multicolumn{3}{|c|}{$\begin{array}{c}\log _{10}\left(x_{S}\right) \text { for } 1.0 \mathrm{M} \\
\text { LiTFSI }\end{array}$} \\
\hline & & exp. & A & B & & exp. & A & B \\
\hline PC & 1.26 & -4.0 & -3.4 & -3.7 & 0.63 & -4.3 & -4.0 & -4.0 \\
\hline DMSO & 3.85 & -3.6 & -2.3 & -3.9 & 1.93 & -3.9 & -3.8 & -4.4 \\
\hline DMF & 5.90 & & -2.2 & -2.8 & 2.60 & -3.7 & -3.5 & -3.6 \\
\hline DME & 8.96 & -3.0 & -2.5 & -2.7 & 3.99 & -3.4 & -3.3 & -3.3 \\
\hline G2 & 9.51 & -2.9 & -2.3 & -2.7 & 3.88 & -3.3 & -3.2 & -3.2 \\
\hline \multirow[t]{2}{*}{ Pyr } & 28.01 & -2.7 & -2.2 & -2.3 & 15.91 & -2.9 & -2.9 & -2.8 \\
\hline & RMSD & & 0.8 & 0.4 & & & 0.2 & 0.3 \\
\hline
\end{tabular}

The solubility of cyclo- $S_{8}$ increases as a function of temperature for all organic solvents and electrolytes, showing the process of sulfur dissolution to be endothermic (Figure 3 and $4-$ using the lowest RMSD solvation approach for each).

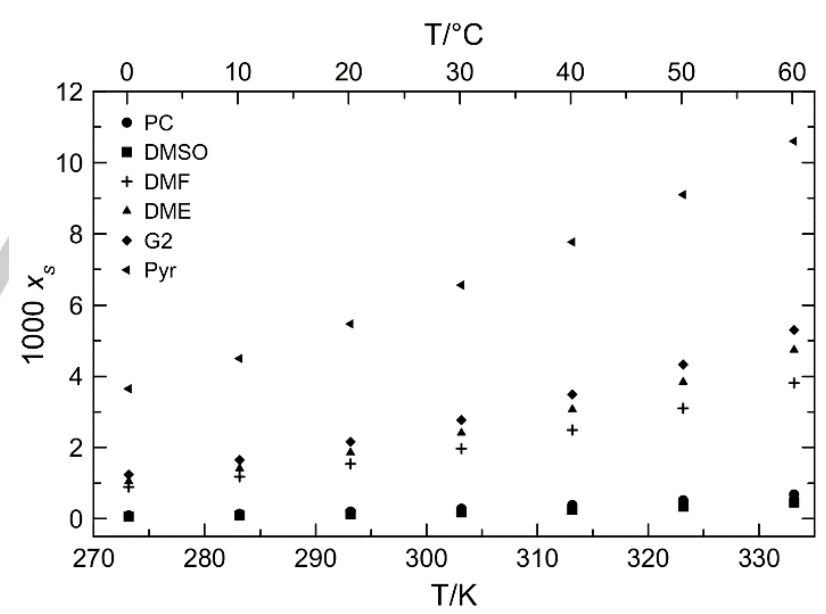


Figure 3. Solubility of cyclo-S $\mathrm{S}_{8}$ in various $0.1 \mathrm{M} \mathrm{LiTFSI} \mathrm{electrolytes} \mathrm{as} \mathrm{a} \mathrm{function}$ of temperature according to the explicit (B) solvation approach.

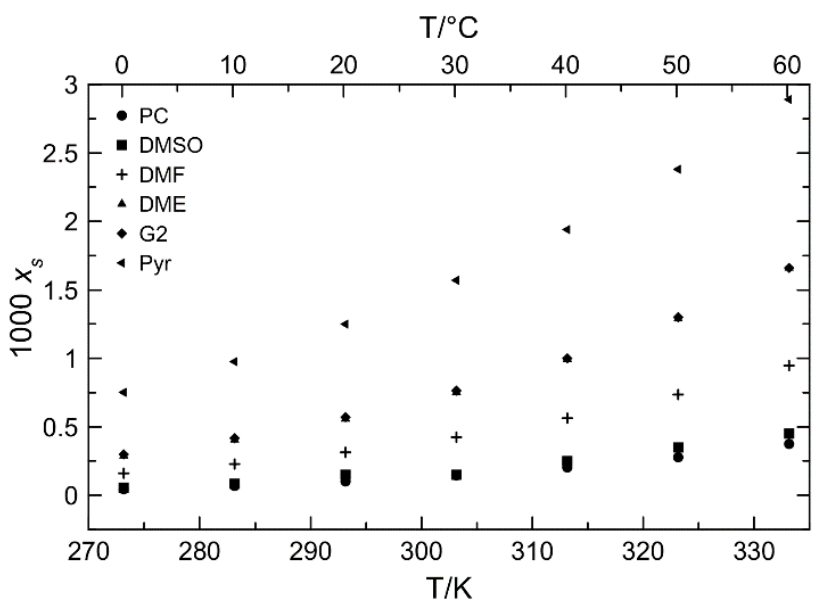

Figure 4. Solubility of $c y c l o-S_{8}$ in various $1.0 \mathrm{M} \mathrm{LiTFSI} \mathrm{electrolytes} \mathrm{as} \mathrm{a} \mathrm{function}$ of temperature according to the implicit $(A)$ solvation approach.

The solubility trend is: $\mathrm{Pyr}>\mathrm{DME} \approx \mathrm{G} 2>\mathrm{DMF}>\mathrm{PC} \approx \mathrm{DMSO}$. It is also clear from Figure 3 and 4 that a higher LiTFSI concentration reduces the sulfur solubility.

\section{Ternary electrolytes}

The cyclo-S $\mathrm{S}_{8}$ solubility was here calculated for a set of ternary electrolytes: $1 \mathrm{M}$ LiTFSI in TMS:DME with different volumetric ratios $(9: 1 \rightarrow 6: 4)$. Again, both the $(A)$ and $(B)$ solvation approaches were used. For the $\mathrm{Li}^{+}-\mathrm{TMS}, \mathrm{Li}^{+}-\mathrm{DME}$ and $\mathrm{Li}^{+}-\mathrm{TFSI}$ clusters the mole fractions were recalculated based on the contact statistics calculation (Table 4). The $\mathrm{Li}^{+}$cation is preferentially contacted by TMS, but with increasing DME content the probability of $\mathrm{Li}^{+}-\mathrm{DME}$ contacts increases, whereas the $\mathrm{Li}^{+}-\mathrm{TFSI}$ contacts remain rather constant for all systems.

Table 4. Contact probability for $\mathrm{Li}^{+}$in the ternary electrolytes.

\begin{tabular}{lllll} 
& $9: 1$ & $8: 2$ & $7: 3$ & $6: 4$ \\
\hline TMS & 0.87 & 0.74 & 0.65 & 0.57 \\
DME & 0.05 & 0.15 & 0.23 & 0.31 \\
TFSI & 0.08 & 0.11 & 0.12 & 0.12 \\
\hline
\end{tabular}

Since the exact densities of the different ternary electrolytes are unknown, the initial mole fractions of TMS, DME, $\mathrm{Li}^{+}$, and TFSI were calculated assuming densities of 1.5 and $1.2 \mathrm{~g} \mathrm{~cm}^{-3}$ and the resulting logarithmic mole fraction solubilities were averaged (see experimental section).

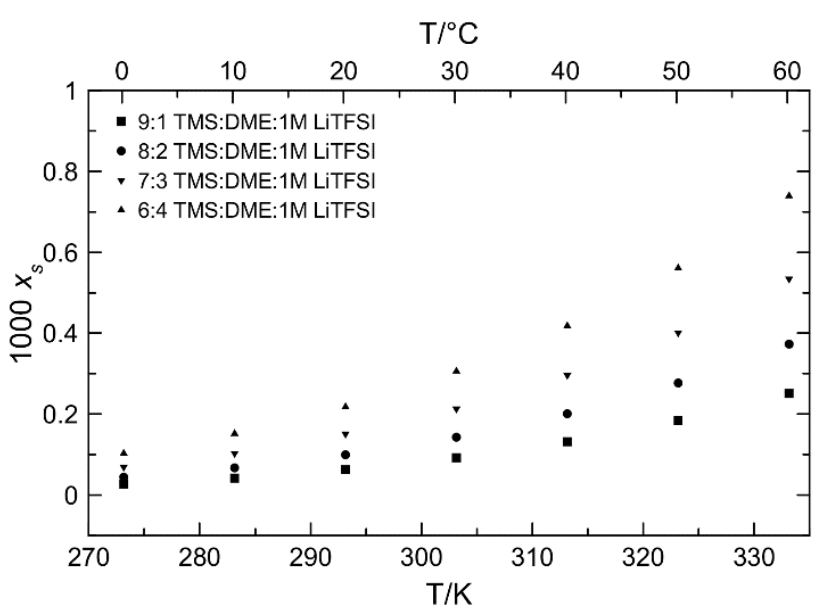

Figure 5. Solubility of cyclo-S $\mathrm{S}_{8}$ in $1.0 \mathrm{M}$ LiTFSI in TMS:DME as a function of temperature according to the implicit $(\mathrm{A})$ solvation approach.

Just as for the binary electrolytes, the two approaches have similar accuracy for the solubility prediction with RMSDs of 0.4 and $0.5 \log$ units, respectively (Table 5 ), and the solubility of cyclo- $_{8}$ increases with temperature, hence an endothermic dissolution process (Figure 5). With increasing content of DME the solubility of $c y c l o-S_{8}$ increases gradually, a trend even more evident for elevated temperatures.

Table 5. Comparison of experimental[ ${ }^{[6]}$ (exp.) sulfur and calculated cyclo-S 8 solubilities in ternary $1.0 \mathrm{M} \mathrm{LiTFSI} \mathrm{in} \mathrm{TMS:DME} \mathrm{electrolytes} \mathrm{using} \mathrm{the} A$ and B solvation approaches.

\begin{tabular}{ccccc}
\hline & & \multicolumn{3}{c}{$\log _{10}\left(x_{S}\right)$ for $1.0 \mathrm{M} \mathrm{LiTFSI}$} \\
\cline { 3 - 5 } TMS:DME & solu./mM & exp. & A & B \\
\hline $9: 1$ & 2.42 & -3.7 & $-4.3( \pm 0.1)$ & $-4.3( \pm 0.0)$ \\
$8: 2$ & 3.11 & -3.6 & $-4.1( \pm 0.1)$ & $-4.1( \pm 0.0)$ \\
$7: 3$ & 3.27 & -3.5 & $-3.9( \pm 0.1)$ & $-3.9( \pm 0.0)$ \\
$6: 4$ & 3.50 & -3.5 & $-3.7( \pm 0.1)$ & $-3.8( \pm 0.1)$ \\
\hline RMSD & & & 0.4 & 0.5 \\
\hline
\end{tabular}

\section{Thermodynamic analysis}

For a better understanding of the dissolution process and its driving forces, a thermodynamic analysis was made in more detail. By plotting the computed solubilities as $\ln \left(x_{S}\right)$ against $1 / T$, the dissolution enthalpy $\left(\Delta_{\text {sol }} H\right)$ and entropy $\left(\Delta_{\text {sol }} S\right)$ can be obtained from the slope and intercept, respectively, using the van't Hoff equation ${ }^{[27,28]}$

$\ln \left(x_{S}\right)=-\frac{\Delta_{s o l} H}{R} \cdot \frac{1}{T}+\frac{\Delta_{s o l} S}{R}$ 
where $\mathrm{R}$ is the universal gas constant. Accordingly, the Gibbs free energy of dissolution $\left(\Delta_{\text {sol }} G\right)$ can be obtained using the mean temperature $\left(T_{\text {mean }}\right)$ of the investigated temperature range $(303.15 \mathrm{~K}):[28]$

$\Delta_{\text {sol }} G=\Delta_{\text {sol }} H-T_{\text {mean }} \Delta_{\text {sol }} S$

To compare the relative contributions of enthalpy $\left(\% \xi_{H}\right)$ and entropy $\left(\% \xi_{T S}\right)$ to the Gibbs free energy of dissolution for the process, the following equations were used:[28,29]

$\% \xi_{H}=100 \times \frac{\left|\Delta_{s o l} H\right|}{\left|\Delta_{s o l} H\right|+\left|T \Delta_{s o l} S\right|}$

$\% \xi_{T S}=100 \times \frac{\left|T \Delta_{s o l} S\right|}{\left|\Delta_{\text {sol }} H\right|+\left|T \Delta_{\text {sol }} S\right|}$

All resulting thermodynamic data from eqs. 2-5 are listed in Table 6. During the dissolution process, solvent-solvent and solutesolute interactions are broken and solvent-solute interactions are formed. The positive values for $\Delta_{\text {sol }} S$ indicate an overall increase in the degree of freedom, but with a contribution of $\% \xi_{H}>80 \%$ for all investigated solutions, the dissolution process of elemental sulfur is clearly enthalpy-driven, but endothermic $\left(\Delta_{\text {sol }} H>0\right)$ and non-spontaneous $\left(\Delta_{s o l} G>0\right)$. As a result and in accordance to the calculated absolute solubilities of cyclo- $\mathrm{S}_{8}$, the dissolution process in general is favored in the following order: Pyr > G2 $\approx$ $\mathrm{DME}>\mathrm{DMF}>\mathrm{DMSO} / \mathrm{PC}$ (the order of DMSO and PC varies upon LiTFSI concentration). For the $0.1 \mathrm{M}$ electrolytes lower $\Delta_{\text {sol }} G$ were obtained compared to the neat organic solvents, indicating the addition of LiTFSI to first favor cyclo-S 8 solubility and then inhibit it upon increasing the salt concentration. This might be attributed to the problems associated with computations for diluted electrolytes with COSMO-RS. However, the experimental solubilities ${ }^{[5]}$ for the neat organic solvents (Table 1) and for the 0.1 M LiTFSI electrolytes (Table 3 ) differ only marginally (except for Pyr).

Table 6. Thermodynamic properties of the dissolution process of cyclo-S $\mathrm{S}_{8}$ in selected organic solvents and binary electrolytes at the mean temperature (303.15 K).

\begin{tabular}{ccccccc}
\hline & $\Delta_{s o l} G^{[a]}$ & $\Delta_{\text {sol }} H^{\text {a] }}$ & $\Delta_{\text {sol }} S^{[\mathrm{b}]}$ & $T \Delta_{\text {Sol }} S^{[a]}$ & $\% \xi_{H}$ & $\% \xi_{T S}$ \\
\hline \multicolumn{2}{l}{ organic solvent } & & & & & \\
PC & 21.7 & 26.1 & 14.6 & 4.4 & 86 & 14 \\
DMSO & 23.3 & 27.0 & 12.1 & 3.7 & 88 & 12 \\
DMF & 18.2 & 20.9 & 9.1 & 2.8 & 88 & 12 \\
DME & 17.2 & 21.2 & 13.4 & 4.1 & 84 & 16 \\
G2 & 17.2 & 20.8 & 11.8 & 3.6 & 85 & 15 \\
Pyr & 12.6 & 13.3 & 2.4 & 0.7 & 95 & 5 \\
DMK & 18.2 & 23.3 & 16.9 & 5.1 & 82 & 18 \\
EtOH & 23.9 & 26.8 & 9.5 & 2.9 & 90 & 10 \\
Hex & 18.6 & 22.6 & 13.5 & 4.1 & 85 & 15
\end{tabular}

0.1 M LiTFSI electrolyte (approach B)

$\begin{array}{lllllll}\text { PC } & 20.6 & 25.0 & 14.6 & 4.4 & 85 & 15\end{array}$

$\begin{array}{ccccccc}\text { DMSO } & 21.7 & 25.4 & 12.3 & 3.7 & 87 & 13 \\ \text { DMF } & 15.7 & 18.3 & 8.6 & 2.6 & 87 & 13 \\ \text { DME } & 15.2 & 19.1 & 12.8 & 3.9 & 83 & 17 \\ \text { G2 } & 14.9 & 18.3 & 11.5 & 3.5 & 84 & 16 \\ \text { Pyr } & 12.7 & 13.4 & 2.4 & 0.7 & 95 & 5\end{array}$

1.0 M LiTFSI electrolyte (approach A)

\begin{tabular}{ccccccc} 
PC & 22.3 & 26.7 & 14.7 & 4.5 & 86 & 14 \\
DMSO & 21.8 & 25.9 & 13.8 & 4.2 & 86 & 14 \\
DMF & 19.6 & 22.3 & 9.0 & 2.7 & 89 & 11 \\
DME & 18.1 & 22.1 & 13.0 & 3.9 & 85 & 15 \\
G2 & 18.1 & 21.6 & 11.6 & 3.5 & 86 & 14 \\
Pyr & 16.3 & 17.0 & 2.2 & 0.7 & 96 & 4 \\
\hline
\end{tabular}

[a] $\mathrm{kJ} \mathrm{mol}^{-1}$ [b] $\mathrm{J} \mathrm{mol}^{-1} \mathrm{~K}^{-1}$

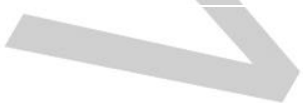

A similar analysis of the ternary $1.0 \mathrm{M}$ LiTFSI TMS:DME electrolytes revealed an inhibiting effect of TMS on the dissolution process of cyclo-S 8 (Table 7).

Table 7. Thermodynamic properties of the dissolution processes of cyclo-S 8 in ternary $1.0 \mathrm{M} \mathrm{LiTFSI} \mathrm{in} \mathrm{TMS:DME} \mathrm{electrolytes} \mathrm{at} \mathrm{the} \mathrm{mean} \mathrm{temperature}$ $(303.15 \mathrm{~K})$

\begin{tabular}{ccccccc}
\hline TMS:DME & $\Delta_{\text {Sol }} G^{[a]}$ & $\Delta_{\text {Sol }} H^{[a]}$ & $\Delta_{S o l} S^{[\mathrm{b}]}$ & $T \Delta_{\text {Sol }} S^{[a]}$ & $\% \xi_{H}$ & $\% \xi_{T S}$ \\
\hline $9: 1$ & 23.8 & 28.4 & 15.3 & 4.6 & 86 & 14 \\
$8: 2$ & 22.7 & 27.3 & 15.0 & 4.5 & 86 & 14 \\
$7: 3$ & 21.7 & 26.1 & 14.7 & 4.5 & 85 & 15 \\
$6: 4$ & 20.8 & 25.2 & 14.5 & 4.4 & 85 & 15 \\
\hline
\end{tabular}

[a] kJ mol ${ }^{-1}[\mathrm{~b}] \mathrm{J} \mathrm{mol}^{-1} \mathrm{~K}^{-1}$

\section{Conclusions}

Using experimentally determined sulfur solubilities as references, the solubility of cyclo-S $S_{8}$ in binary and ternary electrolytes was shown by COSMO-RS simulations to be an endothermic process facilitated by DME as solvent and increased temperature, but decreasing as a function of LiTFSI concentration. From a methodological perspective, for salt concentrations $<1 \mathrm{M}$ the explicit solvation approach performs more accurately, and $>1 \mathrm{M}$ the implicit solvation approach. Overall the computed solubilities differ from the experimental by $<0.4 \log$ units, which is within the experimental error and hence indicates an accurate and reliable performance of the COSMO-RS simulations - rendering the approach here presented useful for screening and understanding at the molecular level. 


\section{Experimental Section}

Examined Compounds: In the COSMOtherm database, cosmo-files for acetonitrile (ACN), acetone (Ace), ethanol (EtOH), n-hexane (Hex), pyridine (Pyr), dimethylformamide (DMF) and dimethylsulfoxide (DMSO) are provided by default. The structures and cosmo-files for cyclo-S, propylene carbonate (PC), dimethoxyethane (DME), diglyme (G2) and tetramethylene sulfone (TMS) were added. For PC, only the $R$-isomer was used. For G2, a conformational search of a G2 dimer was applied by using MOPAC2012 with PM7 ${ }^{[31,32]}$ in a molecular dynamics structure search at $293 \mathrm{~K}$ for $14 \mathrm{ps}$ in $\mathrm{Gabedit}^{[33]}$ (version 2.4.9). The 5 dimeric structures of lowest energy provided $10 \mathrm{G} 2$ conformations, which were added to the COSMSOtherm database. According to the Boltzmann population analysis in COSMOtherm, however, 3/10 G2 conformations represent $99 \%$ of the population, why, in the interest of computational time, the remaining 7 conformations were ignored.

For elemental sulfur, the molar free energy of fusion $\Delta$ fus $G$ is calculated from the corresponding experimental molar heat of fusion $\left(\Delta_{f u s} H\right)$ and melting temperature $\left(T_{m}\right)$ according to

$$
\Delta_{f u s} G(T)=\Delta_{f u s} H \cdot\left(1-T / T_{m}\right)
$$

with $\Delta$ fus $H=17 \mathrm{~kJ} \mathrm{~mol}^{-1}\left(2.1 \mathrm{~kJ} \mathrm{~mol}^{-1}\right.$ for $\left.\mathrm{S}_{1}{ }^{[34]}\right)$ and $T_{m}=388 \mathrm{~K}\left(115^{\circ} \mathrm{C}\right)$ and $T=298.15 \mathrm{~K}$ a value of $3.6 \mathrm{~kJ} \mathrm{~mol}^{-1}$ for $\Delta$ fus $G$ is obtained for the cyclo$\mathrm{S}_{8}$ allotrope.

Computational Methods: Structures were built in the graphical user interface (GUI) of TmoleX 4.1 and the quantum chemical calculations were performed using the TURBOMOLE ${ }^{[35,36]}$ V7.0 program package. Geometries were optimized using the BP86-functional[37,38] and TZVP basis set ${ }^{[39]}$ in gas phase and for the perfect conductor (COSMO: $\varepsilon=\infty$ ). Additionally, a single point calculation at BP86/TZVP//BP86/TZVPD level of theory was performed for gas phase and cosmo phase geometries in order to generate a fine grid cavity surface (FINE) for the molecules which is saved in a cosmo-file. The COSMO-RS calculations were performed using COSMOtherm and the BP_TZVPD_FINE_C30_1501 parametrization via the COSMOtherm $X$ GUI. ${ }^{[40]}$ The solvent screening module was used to determine $\triangle_{\text {fus }} G$ of $c y c / o-S_{8}$, which represents elemental sulfur, according to its solubility in various pure organic solvents. The extended mixtures module was used to compute the intermolecular contact statistics at $273.15 \mathrm{~K}$. The probability, $p_{a b}$, that a segment of molecule $A$ is in contact with a segment of molecule $B$ is given by

$$
p_{a b}=\frac{x_{B} A_{a} A_{b} \gamma_{a} \gamma_{b}}{A_{\text {total }}^{A} A_{\text {total }}} \exp \left(-E_{a b} / k T\right)
$$

The solid-liquid module was used to compute the solubility in various binary and ternary electrolytes in a temperature range from 273.15 to $333.15 \mathrm{~K}\left(0\right.$ to $\left.60^{\circ} \mathrm{C}\right)$ in steps of $10 \mathrm{~K}$. All computations were performed with regard to the mole fraction of the containing species. The mole fractions were calculated according to:

$$
x_{1}=\frac{n_{1}}{\sum n}
$$

The moles $n$ of $\mathrm{Li}^{+}$and TFSI ${ }^{+}$are obtained via

$$
n_{L i+/ T F S I}=c_{e l} \cdot V
$$

where $c_{e l}$ is the concentration of LiTFSI in the electrolyte and $V$ the volume $(1 \mathrm{ml})$. For a solvent $i$, the moles $n_{i}$ were calculated according to:

$$
n_{i}=\frac{\rho_{i} \cdot V \cdot f_{i}}{M_{i}}
$$

where $\rho_{i}, M_{i}$ and $f_{i}$ are the density, the molar mass and the volume fraction of the solvent $i$., respectively. In all binary electrolytes, the density of the organic solvent was used. The densities for neat TMS:DME mixtures was calculated neglecting a potential volume contraction according to:

$$
\rho_{\text {calc }}=f_{T M S} \cdot \rho_{T M S}+f_{D M E} \cdot \rho_{D M E}
$$

The obtained densities are between $1.1 \mathrm{~g} \mathrm{~cm}^{-3}$ for a volumetric ratio of $6: 4$ and $1.2 \mathrm{~g} \mathrm{~cm}^{-3}$ for $9: 1$. To account the increase in density due to addition of LiTFSI, the mole fraction solubilities of cyclo-S $x_{s} x^{1.5}$ and $x_{s}^{1.2}$ were computed assuming a density of 1.5 and $1.2 \mathrm{~g} \mathrm{~cm}^{-3}$ for all ternary electrolytes, respectively. The results obtained for either density were averaged and the standard deviation was calculated.

The root-mean-square deviation (RMSD) was calculated according to:

$$
R M S D=\sqrt{\frac{1}{N} \sum_{i=1}^{N}\left(\log _{10}\left(x_{s, i}^{c a l c}\right)-\log _{10}\left(x_{s, i}^{e x p}\right)\right)^{2}}
$$

where $x_{s, t}$, calc and $x_{s, i}{ }^{\text {exp }}$ are the calculated and experimental mole fraction solubility, respectively, of cyclo-S 8 in solvent $i$.

\section{Acknowledgements}

The European Commission has provided financial support for 'High Energy Lithium Sulfur Cells and Batteries" (HELIS) under research grant agreement No. 666221. We thank Dr. Franck Eckert (COSMOlogic $\mathrm{GmbH} \&$ Co.KG) for valuable discussions.

Keywords: COSMO-RS $\cdot$ Lithium-Sulfur battery $\cdot$ sulfur solubility $\cdot$ electrolyte design $\cdot$ excess properties

[1] M. R. Busche, P. Adelhelm, H. Sommer, H. Schneider, K. Leitner, J. Janek, J. Power Sources 2014, 259, 289-299.

[2] P. Adelhelm, P. Hartmann, C. L. Bender, M. Busche, C. Eufinger, J. Janek, Beilstein J. Nanotechnol. 2015, 6, 1016-1055.

[3] M. Barghamadi, A. S. Best, A. I. Bhatt, A. F. Hollenkamp, M. Musameh, R. J. Rees, T. Rüther, Energy Environ. Sci. 2014, 7, 3902-3920.

[4] J. Scheers, S. Fantini, P. Johansson, J. Power Sources 2014, 255, 204-218.

[5] D. Zheng, X. Zhang, C. Li, M. E. Mckinnon, R. G. Sadok, D. Qu, X. Yu, H.-S. Lee, X.-Q. Yang, D. Qu, J. Electrochem. Soc. 2015, 162, 203-206.

[6] S. Yoon, Y.-H. Lee, K.-H. Shin, S. B. Cho, W. J. Chung, Electrochim. Acta 2014, 145, 170-176.

[7] A. Klamt, J. Phys. Chem. 1995, 99, 2224-2235.

[8] M. Diedenhofen, A. Klamt, K. Marsh, A. Schäfer, Phys. Chem. Chem. Phys. 2007, 9, 4653-4656

[9] T. Banerjee, M. K. Singh, A. Khanna, Ind. Eng. Chem. Res. 2006, 45, 3207-3219.

[10] I. Khan, K. A. Kurnia, T. E. Sintra, J. A. Saraiva, S. P. Pinho, J. A. P. 
Coutinho, Fluid Phase Equilib. 2014, 361, 16-22.

[11]

M. P. Andersson, S. L. S. Stipp, J. Phys. Chem. C 2012, 116

18779-18787.

[12] M. P. Andersson, M. H. M. Olsson, S. L. S. Stipp, Langmuir 2014 , 30, 6437-6445

[13] J. J. Guerard, J. S. Arey, J. Chem. Theory Comput. 2013, 9, 50465058.

[14] A. V. Marenich, J. Ho, M. L. Coote, C. J. Cramer, D. G. Truhlar, Phys. Chem. Chem. Phys. 2014, 16, 15068-15106.

[15] C. Loschen, A. Klamt, J. Pharm. Pharmacol. 2015, 67, 803-811.

[16] C. Loschen, A. Klamt, Pharm. Res. 2016, 1-11.

[17] L. Y. Garcia-Chavez, A. J. Hermans, B. Schuur, A. B. de Haan, Sep. Purif. Technol. 2012, 97, 2-10.

[18] J. Palomar, J. S. Torrecilla, J. Lemus, V. R. Ferro, F. Rodríguez, Phys. Chem. Chem. Phys. 2010, 12, 1991.

M. Gonzalez-Miquel, M. Massel, A. DeSilva, J. Palomar, F.

Rodriguez, J. F. Brennecke, J. Phys. Chem. B 2014, 118, 1151211522.

[20]

V. Govinda, T. Vasantha, I. Khan, P. Venkatesu, Ind. Eng. Chem. Res. 2015, 54, 9013-9026.

[21] A. Klamt, Fluid Phase Equilib. 2016, 407, 152-158.

[22] O. Toure, F. Audonnet, A. Lebert, C.-G. Dussap, Chem. Eng. Res. Des. 2014, 92, 2873-2883.

[23]

O. Toure, F. Audonnet, A. Lebert, C.-G. Dussap, Can. J. Chem. Eng. 2015, 93, 443-450.

[24] J. J. Griebel, R. S. Glass, K. Char, J. Pyun, Prog. Polym. Sci. 2016, 58, 90-125.
B. Meyer, Chem. Rev. 1976, 76, 367-388.

H. J. Kandiner, M. Atterer, L. Gmelin, Schwefel, Springer Berlin Heidelberg, Berlin, Heidelberg, 1974.

[27] L. Song, Y. Gao, J. Gong, J. Chem. Eng. Data 2011, 56, 25532556.

[28] X.-Y. Qing, H.-L. Fu, G. Shu, M.-J. Liu, J.-Y. Zhou, W.-B. Wu, Y.-L. Zhang, J.-J. Zhou, J. Chem. Eng. Data 2015, 60, 1629-1633.

[29] G. L. Perlovich, S. V. Kurkov, A. Bauer-Brandl, Eur. J. Pharm. Sci. 2003, 19, 423-432.

[30] K. A. Kurnia, J. A. P. Coutinho, Ind. Eng. Chem. Res. 2013, 52, 13862-13874.

[31] J. D. C. Maia, G. A. Urquiza Carvalho, C. P. Mangueira, S. R. Santana, L. A. F. Cabral, G. B. Rocha, J. Chem. Theory Comput. 2012, 8, 3072-3081.

[32] J. J. P. Stewart, n.d.

[33] A.-R. Allouche, J. Comput. Chem. 2011, 32, 174-182.

[34] D. R. Lide, Handb. Chem. Phys. 2012, 93rd editi, 2664.

[35] R. Ahlrichs, M. Bär, M. Häser, H. Horn, C. Kölmel, Chem. Phys. Lett. 1989, 162, 165-169.

\section{[36] 2015}

[37] A. D. Becke, Phys. Rev. A 1988, 38, 3098-3100.

[38] J. P. Perdew, Phys. Rev. B 1986, 33, 8822-8824.

[39] A. Schäfer, C. Huber, R. Ahlrichs, J. Chem. Phys. 1994, 100, 5829.

[40] F. Eckert, A. Klamt, 2015. 
Entry for the Table of Contents (Please choose one layout)

Layout 1:

\section{FULL PAPER}

Using experimentally determined sulfur solubilities as references, the solubility of cyclo-S $\mathrm{S}_{8}$ was predicted in binary and ternary electrolytes by COSMO-RS computations for the first time. Presented results prove this method to be a tool for exploring novel Li-S battery electrolytes and enhanced understanding at the molecular level.
Steffen Jeschke* and Patrik Johansson

Page No. - Page No.

Predicting solubility of sulfur: A COSMO-RS based approach to investigate electrolytes for $\mathrm{Li}-\mathrm{S}$ batteries 